\title{
Spectrofluorimetric analysis in vivo: applications to kinetics of ALA induced fluorescence
}

\author{
V. VONARX, S. CORDEL, P. LENZ*, M.T. FOULTIER, R. POTTIER ${ }^{* *}$, J. KENNEDY** and \\ T. PATRICE
}

\author{
Dpt Laser, Hôpital Laennec, 44035 Nantes, France \\ * U 281 INSERM, 69424 Lyon, France ${ }^{* *}$ RMC, K7K 5LO Kingston, Canada
}

\section{INTRODUCTION}

Standard forms of PDT involve the administration of an endogenous photosensitizer. This photosensitizer accumulates preferentialiy in malignant tissues ${ }^{1}$ and exposure to appropriate light wavelengths induces a phototoxic effect by producing active molecular species such as singlet oxygen $\left(\mathrm{O}_{2}\right)$ and free radicals 2 . The advantage of photodynamic therapy (PDT) over conventional therapy is its dual selectivity ${ }^{3}$. First, increased selectivity is obtained by localization of the photosensitizer in tumor, and secondly toxicity is limited to the irradiated area, thereby avoiding serious damage to adjacent normal tissue. However, it remains difficult to document this selectivity routinely in patients. Selectivity is not likely to occur in all patients since tumors are heterogeneous and could also be transient. The capability of monitoring sensitizer concentration in tissues is thus of considerable importance. If that compound can be induced to fluoresce when exposed to photons of appropriate wavelength, then it may be possible to identify the compound of interest because of its characteristic fluorescence emission or excitation spectrum and then to follow its synthesis and/or degradation in vivo by measuring changes in fluorescence intensity. In vivo spectrofluorometry laser technique appears to have significant advantages over other methods. It is highly sensitive and can record complete fluorescence emission spectra so rapidly that it is now feasible to obtain a series of such spectra in anesthetized or non-anesthetized animals. An alternative approach to sensitizer administration for PDT is to achieve photosensitizer synthesis in situ using a precursor that is either preferentially localized or preferentially converted to active photosensitizer by target tissue or cells. ALA is an endogenous substance which is converted to heme via PpIX, an effective and well-known photosensitizer associated with the extreme skin phototoxicity seen in patients with porphyria $4,5,6$. The present study was designed to investigate the possibility of monitoring $\mathrm{PpIX}$ fluorescence by the mean of a fiberoptic laser spectrofluorimeter designed in collaboration with INSERM U.281 (P.Lenz) after ALA injection We also tried to enhance fluorescence by modifying PplX concentration using the iron chelator desferrioxamine, also known as Desferal ${ }^{R} 7$, as well as EDTA an other metal chelator.

\section{MATERIALS AND METHODS}

2.1 Fluorescence spectrometer

$488 \mathrm{~nm}$ light from an argon laser was used to excite fluorescence which was then.attenuated by reflection on a glass plate and fed into a 600 um core plastic-clad silica fiber with perpendicular polished endfaces by means of a small $45^{\circ}$ metal mirror and an achromatic lens. The distal fiber end was brought into direct contact with the target tissue. This system provides simultaneous delivery of excitation light and gathering of fluorescence light. Light leaving the fiber is imaged onto a glass fiber bundle (ORIEL 77402) through the above-mentioned achromatic lens, a second achromatic lens located behind the metal mirror and a high-pass filter (SCHOTT OG 530) which virtually eliminates backscattered laser light. The fiber bundle converts the circular beam into a rectangular one. Thus convenient spectral resolution is obtained when this system is coupled to the monochromator (JOBIN YVON CP 200). Spectra are recorded using a cooled 1024-element diode array and a 386 computer (with JOBIN YVON QUIKVIEW software). . The $488 \mathrm{~nm}$ line of an argon ion laser was used for excitation. Recording of a single spectrum took $20 \mathrm{~ms}$, but we integrated over $3 \mathrm{~s}$. Power density was $2 \mathrm{~mW} \mathrm{~cm}^{-2}(488 \mathrm{~nm})$. Light (excitation and reception) was then transmittted through a $600 \mathrm{um}$ silica silicon step index optic fiber.

\subsection{Animals}

Six-weeks-old NMRI hairless male nude mice (CERJ) weighing approximatively $30 \mathrm{~g}$ were used in this experiment. HT 29 tumor (human colic adenocarcinoma) was injected sub-cutaneously $(0,2 \mathrm{ml}$ of crushed tumor in solution in culture medium). All mice received a tumor graft [8]. Tumors were allowed to grow for 12 days until the experiment was performed. At that time, their mean diameter was $10 \mathrm{~mm}$ (SD $+/-2 \mathrm{~mm})$.

2.3. Chemicals

5-aminolevulinic acid (ALA) was obtained from Sigma Chemical Company (UK). Solutions containing 30,60 or $90 \mathrm{mg}$ of ALA per milliliter of isotonic saline were prepared immediately before use. $0,25 \mathrm{ml}$ was injected intraperitoneally in order to reach final mouse concentrations of 250,500 and $750 \mathrm{mg} / \mathrm{kg}$ of body weight. All mice were kept in the dark until spectrofluorometry was performed. Desferrioxamine . was purchased in the lyophilized form of desferrioxamine mesylate (Desferal [DFO]) from CIBA-Geigy (Basel, 
Switzerland). This compound was administered subcutaneously to obtain concentrations of 20 and 40 $\mathrm{mg} / \mathrm{kg}$ of body weight. EDTA was obtained from Grosseron (GROSSERON S.A., Nantes, France) and administered subcutaneously to obtain concentrations of 10 and $20 \mathrm{mg} / \mathrm{kg}$ of body weight.

\subsection{Method}

Kinetic fluorometric studies were performed after administration of etheroxide inhalant at intervals of 0 ; $30 \mathrm{~min}$; $60 \mathrm{~min}$; $90 \mathrm{~min} ; 2 \mathrm{~h} ; 3 \mathrm{~h} ; 4 \mathrm{~h}$ and $6 \mathrm{~h}$ and as well as in different tissues. We carried out measurements through the optic fiber placed directly in contact with the target. DFO and EDTA were injected subcutaneously 4 hours before ALA administration. Two, three and five hours after ALA injection, spectra were recorded directly in tissues. Thus, DFO or EDTA was injected at least 6 hours before spectrum recording. Muscle, tumor, liver, gall bladder, urinary bladder, kidney, heart and brain were successively studied. The same experimental conditions used to obtain sample material, anesthesic techniques and darkness were applied to the different groups. The fluorescence emission spectra of treated or untreated mouse tissue were recorded on 3 separate occasions in order to estimate mouse-to-mouse variation. 3. RESULTS

3.1. In vivo PplX fluorescence spectra after ALA injection

Administration of a $250 \mathrm{mg} / \mathrm{kg}$ ALA solution to mice resulted in an increase in tissue fluorescence, with emission maxima at $632 \mathrm{~nm}$ and $702 \mathrm{~nm}$. All mice produced comparable PplX emission spectra for all tissues studied. The intensity of this fluorescence increased for approximatively $180 \mathrm{~min}$ in lung and kidney but for only $120 \mathrm{~min}$ in muscle and gall bladder gradually decreasing over a 3 -h period.

Figure 1 shows the spectral modifications of fluorescence in HT 29 adenocarcinoma (A) or normal liver (B) as a function of time. PplX fluorescence was maximal after $180 \mathrm{~min}$ in liver but only after $90 \mathrm{~min}$ in tumors.

in addition, we noted an increased fluorescence emission at $545 \mathrm{~nm}$ in liver, gall bladder and urinary bladder. This emission wavelength, typical of metabolized porphyrins such as bilirubin, was present in controls but increased after ALA injection. In controls receiving no ALA, $545 \mathrm{~nm}$ fluorescence was $30 \mathrm{cts} / \mathrm{s}$ in gall bladder and urinary bladder but increased after $60 \mathrm{~min}$ (gall bladder) and $360 \mathrm{~min}$ (urinary bladder) to a mean value of 168 and $101 \mathrm{cts} / \mathrm{s}$ respectively. $632 \mathrm{~nm}$ fluorescence was also found in the urinary bladder less than 240 min after ALA injection but then decreased (Fig 1C).
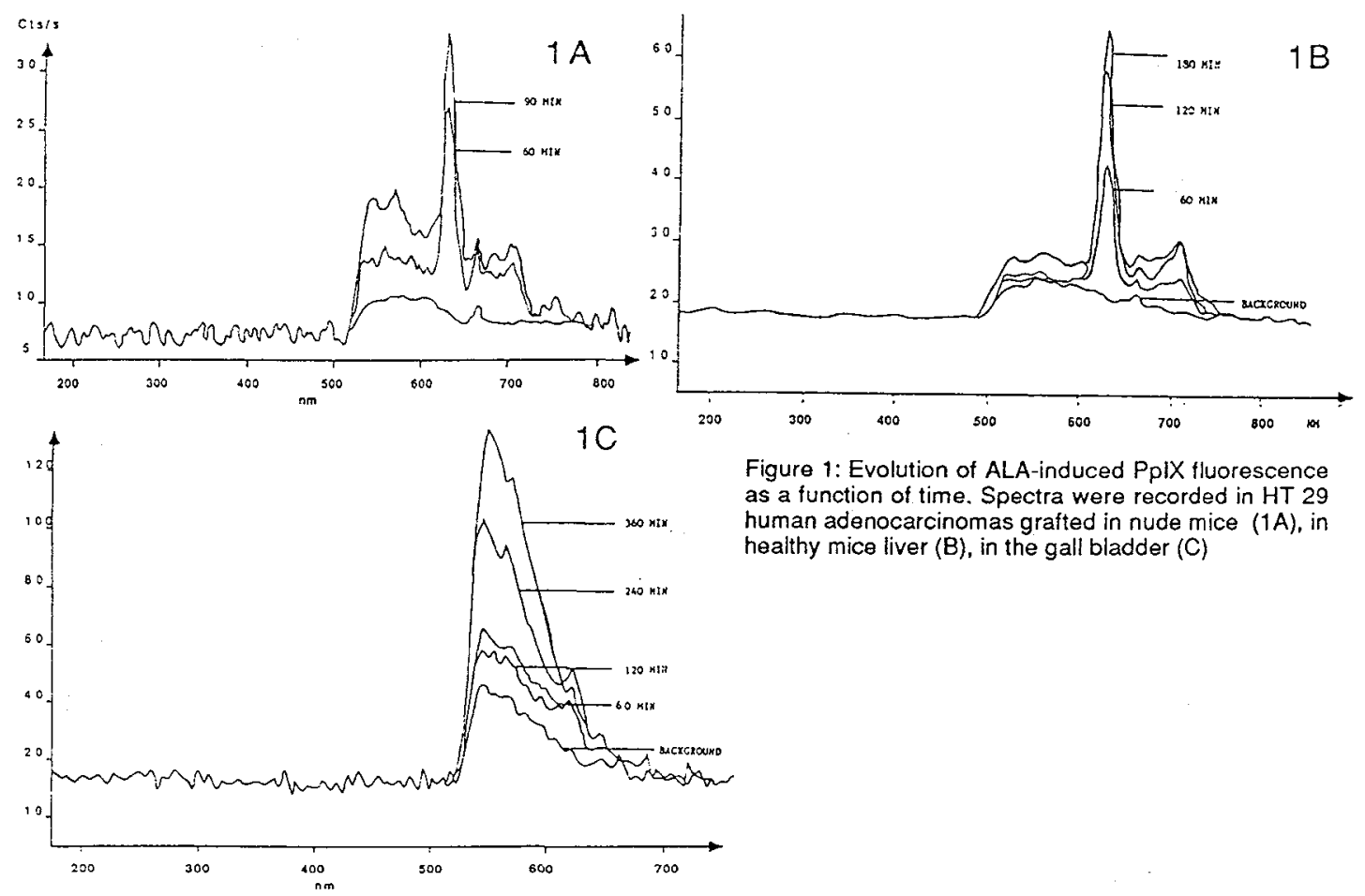

Figure 1: Evolution of ALA-induced PplX fluorescence as a function of time. Spectra were recorded in HT 29 human adenocarcinomas grafted in nude mice $(1 \mathrm{~A})$, in healthy mice liver $(B)$, in the gall bladder $(C)$

3.2. Changes in PpIX fluorescence after ALA injection

Tmax and fluorescence intensities differed from one organ to another. In addition, in some organs fluorercence increased and decreased in several phases. In heart, brain and lung, fluorescence intensity was relatively low (about $10-25 \mathrm{cps} / \mathrm{s}$ ) but in elimination organs the fluorescence level was significantly higher, i.e. gall bladder with a recording of about $610 \mathrm{cps} / \mathrm{s}$. 
TABLE 1 : Evolution of PPIX after ALA injection

\begin{tabular}{|c|c|c|c|c|c|}
\hline TISSUES & Tmax FLuO & $\underset{I^{*}}{\text { CONTROL }}$ & INTENSITY & $y x^{*}$ & \\
\hline $\begin{array}{l}632 \mathrm{~nm} \\
\text { TUMOR }\end{array}$ & $90 \mathrm{~min}$ & 3 & 31 & 90,33 & \\
\hline MUSCLE & $120 \mathrm{~min}$ & 2 & 11,66 & 5,83 & \\
\hline LIVER & $60.180 \mathrm{~min}$ & 7 & 82,25 & 11,75 & \\
\hline GALL BLADDER & $120 \mathrm{~min}$ & 9 & 610 & 67,8 & $\begin{array}{l}T \text { MAX :TIME AT WHICH FLUORESCENCE INTENSTYY IS MAXIMAL } \\
\text { AFTER ALA INJECTION }\end{array}$ \\
\hline BLADDER & $\begin{array}{l}60-120-240 \\
300 \mathrm{~m} \text { in }\end{array}$ & g & 40 & 4,44 & $\begin{array}{l}\text { I* : BACKGROUNO INTENSITY IN TISSUES } \\
\text { I/" : INTENSITY IN TISSUE AFTER CHEMICAL ADMINISTRATION } \\
\text { TO BACKGROUND INTENSITY WAS STUDIED IN TISSUE. }\end{array}$ \\
\hline KIDNEY & $180 \mathrm{~min}$ & 4 & 31,38 & 7,84 & \\
\hline BRAIN & $120 \mathrm{~min}$ & 3,5 & 13 & 3,71 & \\
\hline MEART & $60-120 \mathrm{~min}$ & 7 & 11 & 1,57 & \\
\hline LUNG & $180 \mathrm{~min}$ & 7 & 24 & 3,43 & \\
\hline $\begin{array}{l}545 \mathrm{~nm} \\
\text { GALL BLADDER }\end{array}$ & $60 \mathrm{~min}$ & 30 & 168,5 & 5,62 & \\
\hline BLADDER & $60.360 \mathrm{~min}$ & 29 & 101 & 3,48 & \\
\hline
\end{tabular}

\subsection{Influence of DFO on ALA-induced PpIX fluorescence}

Administration of 20 or $40 \mathrm{mg} / \mathrm{kg}$ DFO alone did not enhance background tissue fluorescence. DFO administered before ALA induced different effects on PpIX fluorescence changes. Tissues can again be divided into 3 groups (Table 2). In the first group. PpIX fluorescence intensity increased in HT 29 adenocarcinoma, muscle, heart and gall bladder, but the time required to reach maximum fluorescence was the same in these tissues as in mice injected withALA alone. In the second group. DFO 20 or $40 \mathrm{mg} / \mathrm{kg}$ induced only slight changes in ALA-stimulated porphyrin production which was even somewhat decreased compared, to that of controls. However, in liver there was a shorter interval after ALA administration before maximal fluorescence was reached. In kidney and brain, on the contrary, Tmax was shifted to $5 \mathrm{~h}$ with DFO instead of $3 \mathrm{~h}$ as in controls. In the urinary bladder, both Tmax and fluorescence intensity were changed for DFO $40 \mathrm{mg} / \mathrm{kg}$. The PpIX fluorescence intensity induced by ALA increased after DFO administration, and there was a shortening of the Tmax shifting from $5 \mathrm{~h}$ to $3 \mathrm{~h}$. After DFO $20 \mathrm{mg} / \mathrm{kg}$, fluorescence intensity increased, but the kinetic was similar to that of controls.

\begin{tabular}{|c|c|c|c|c|c|c|c|c|c|}
\hline DFO & $\operatorname{Tmax}$ & Ima & \|\|$^{*}$ & $T \max$ & $\begin{array}{l}20 \mathrm{mg} / \\
\text { Imax }\end{array}$ & $\mid / I^{*}$ & $T \max$ & $\underset{I \max }{40 \mathrm{mg} / \mathrm{kg}}$ & $\mathbf{l} / \mathbf{l}^{*}$ \\
\hline \multicolumn{10}{|l|}{ GROUP 1} \\
\hline TUMOA & $2 h$ & 29,33 & 9,78 & $2 h$ & 41,5 & 13,83 & $2 h$ & 34 & 11,33 \\
\hline MUSCLE & $2 \mathrm{~h}$ & 14 & 7 & $2 h$ & 14,33 & 7,165 & $2 h$ & 17,5 & 8,75 \\
\hline HEART & $2 \mathrm{~h}$ & 11 & 1,57 & $2 \mathrm{~h}$ & 20 & 2,86 & $2 \mathrm{~h}$ & 20 & 2,86 \\
\hline GALL BLADDER & $2 h$ & 610 & 67,77 & $2 h$ & 987 & 109,66 & $2 h$ & 390 & 43,33 \\
\hline \multicolumn{10}{|l|}{ GROUP 2} \\
\hline LIVER & $3 \mathrm{~h}$ & 106 & 15,14 & $2 \mathrm{~h}$ & 72,66 & 10,38 & $2 \mathrm{~h}$ & 60,33 & 8,62 \\
\hline KIDNEY & $3 \mathrm{~h}$ & 38 & 9.5 & $5 \mathrm{~h}$ & 31,5 & 7,87 & $5 \mathrm{~h}$ & 32,5 & 8,125 \\
\hline BRAIN & $3 h$ & 20 & 5,71 & $5 \mathrm{~h}$ & 5 & 1,43 & $5 \mathrm{~h}$ & 4,5 & 1,28 \\
\hline \multicolumn{10}{|l|}{ GROUP 3} \\
\hline BLADDER & $5 \mathrm{~h}$ & 40 & 4,44 & $5 \mathrm{~h}$ & 84 & 9,33 & $3 h$ & 71 & 7,89 \\
\hline
\end{tabular}

\subsection{Influence of EDTA on ALA-induced PpiX fluorescence.}

Administration of 10 or $20 \mathrm{mg} / \mathrm{kg}$ EDTA alone did not enhance background tissue fluorescence. Tissues can be separated into 3 groups in terms of EDTA effect

$:$ In the first group. EDTA decreased 
PpIX fluorescence intensity to a non-significant level. In liver, fluorescence intensity was lower with EDTA and ALA than with ALA alone. However, fluorescence remained easily detectable. In liver and gall bladder, although Tmax were unchanged, maximum fluorescence decreased for $10 \mathrm{mg} / \mathrm{kg}$ but increased for 20 $\mathrm{mg} / \mathrm{kg}$. Tumor tissues showed a Tmax identical to controls but minimum fluorescence with $10 \mathrm{mg} / \mathrm{kg}$ and then increased amounts. EDTA decreased Tmax and fluorescence intensity in kidney and urinary bladder. 4. DISCUSSION

We designed a laser spectrofluorometer to measure tissue sensitizer concentration since PDT efficacy is at least partly concentration-related. A new means of PDT sensitization was studied, involving administration of ALA to induce endogenous porphyrin synthesis. Kinetic studies of porphyrin biosynthesis in mouse tissues are presented as examples of the potential usefulness of the spectrofluorometer.

As ALA is non- or weakly fluorescent, the emission spectrum is related to the fluorescent material that appears in the mouse tissues after systemic administration which is thought to be PpIX.

This study showed a strong correlation between the intensity and location of ALA-induced PpIX fluorescence. In the absence of significant concentrations of quenching substances, that cell will begin to develop PplX fluorescence and also will become photosensitized [11]. However, tissues such as liver may contain a high concentration of PpIX fluorescence but emit little because of internal quenching of the fluorescence. All of this indicates why fluorescence depends greatly on the organ involved as well as the beside ALA dose or the internal after injection.

We investigated the distribution of ALA-induced porphyrin fluorescence at various intervals after introduction of ALA, both in normal and tumor tissue in the mouse. Divaris et al. ${ }^{9}$ have shown that the intensity of ALA-induced PpIX fluorescence in tissue after systemic administration of ALA to mice is correlated with the amount of phototoxic damage. Maximum fluorescence is rapidly obtained with nearmaximal tissue levels of PplX reached at 2-3h. We also demonstrated a significant advantage of ALA-induced porphyrin over conventional sensitisers in that the sensitization level returns to nearly background fluorescence after 6 hours, except in urinary bladder, gall bladder and liver.

Modulation of ALA-induced porphyrin synthesis is also of interest as a means of eventually increasing PpiX concentration in tumors. Since desferrioxamine (DFO) is used as a therapeutic iron chelator in humans, we investigated whether tetrapyrrol synthesis may be stimulated in mammalian cells by DFO iron trapping. DFO is reported to be a very potent stimulator of ALA-driven photosensitization in vitro. In our study, DFO or EDTA administration before ALA injection induced no significant increase in PplX fluorescence but rather an inhibitory action on ALA-induced PpIX fluorescence. In addition, it seemed to us that DFO and EDTA induced modifications in elimination pathways of endogenously synthetized porphyrins.

\section{REFERENCES}

1. T. Patrice, V. Praloran, M. F. Le Bodic and L. Le Bodic, Experimental aspects of in vitro and in vivo photochemotherapy, Biochimie, 68 923-926, 1986.

2. K. R. Weishaupt, C. J. Gomer and T. J. Dougherty, Identification of singlet oxygen as the cytotoxic agent in photoinactivation of murine tumor, Cancer Res., 36 2326-2329, 1976.

3. T. Patrice, Challenges for PDT in treatment of gastrointestinal tumors, Photochem. Photobiol., B. 9 372374, 1991.

4. R. H. Pottier, Y. F. A. Chow, J. P. La Plante, T. G. Truscott, J. C. Kennedy and L. A. Beiner, Non-invasive technique for obtaining fluorescence excitation and emission spectra in vivo, Photochem. Photobiol., 44 $679-687,1986$.

5. J. C. Kennedy, R. H. Pottier and D. C. Pross, Photodynamic therapy with endogenous protoporphyrin IX: basic principles and present clinical experience, Photochem. Photobiol. B: Biol., 6 143-148, 1990.

6. B. A. Goff, R. Bachor, N. Kolias and T. Hasan, Effects of photodynamic therapy with topical application of 5aminolevulinic acid on normal skin of hairless guinea pigs, Photochem. Photobiol. B: Biol., 15 239-251, 1992.

7. B. Ortel, A. Tanew and H. Hönigsmann, Lethal photosensitization by endogenous porphyrins of PAM cells-modification by desferrioxamine, Photochem.Photobiol. B,17 273-278, 1993.

8. T. Patrice, M. T. Foultier, S. Yacato, M. C. Douet, F. Maloisel and L. Le Bodic, Endoscopic photodynamic therapy with hematoporphyrin derivative in gastroenterology, Photochem.Photobiol., B. $6157-165,1990$.

9. D. X. G. Divaris, J. C. Kennedy and R. H. Pottier, Phototoxic damage to sebaceous glands and hair follicles of mice after systemic administration of 5-aminolevulinic acid correlates with localized protoporphyrin IX fluorescence, Am. J. Path., 136 891-897, 1990.

10. J. Bedwell, A. J. Mac Robert, D. Phillips and S. G. Bown, Fluorescence destribution and photodynamic effect of ALA-induced PPIX in the DMH rat colonic tumour model, Br. J. Cancer, 65 818-824, 1992. 